\title{
La fracción evaporativa en diferentes regiones agrícolas y naturales del centro- noroeste de la Argentina
}

\author{
Luna Toledo, E.S.; P.I. Figuerola, E.G. Abril y A.C. Ravelo
}

\begin{abstract}
RESUMEN
La fracción evaporativa (FE) permite cuantificar la porción de la energía disponible usada en el proceso de evapotranspiración. Se obtuvo la distribución espacial de la FE para cinco tipos de cobertura: árboles, cultivos, pastizales, arbustos y suelo desnudo de cinco ecorregiones del centronoroeste de la Argentina, usando productos del sensor MODIS-TERRA. FE se calculó mediante el método triangular según la relación entre la fracción de la cobertura de suelo $(\mathrm{Fr})$ y la temperatura de superficie $\left(\mathrm{T}_{\mathrm{s}}\right)$. Ambas variables fueron correlacionadas para cada tipo de cobertura por ecorregión. Fr y Ts están inversamente asociadas, y diferentes coberturas por ecorregiones mostraron cambios en la pendiente de la recta entre ambas variables debido a cambios en el porcentaje y el tipo de cobertura, en los gradientes pluviométricos y en la estructura de la vegetación. Los valores medios de Fr y FE por ecorregiones y tipo de cobertura fueron procesados mediante el análisis de la varianza y por comparaciones múltiples de Fisher. Existen diferencias estadísticas significativas en las variables Fr y FE según el tipo de cobertura, y la ecorregión y la relación entre Fr y FE no es lineal. Finalmente, se generó un mapa con la distribución espacial de la FE.
\end{abstract}

Palabras clave: fracción evaporativa, ecorregiones, MODIS, heterogeneidad.

Luna Toledo, E.S.; P.I. Figuerola, E.G. Abril and A.C. Ravelo, 2013. Evaporative fraction in different agriculture and natural regions of center-northwestern Argentina. Agriscientia 30 (1): 13-24

\section{SUMMARY}

The evaporative fraction (FE) allows quantifying the portion of the available energy used in the process of evapotranspiration. The spatial distribution of the FE is obtained for five types of land covers: Trees, crops, grassland, shrubs and bare soil in five eco-regions of Central-North Western Argentina using MODIS sensor products. FE was calculated using a triangle method for the relation between the fraction of the soil cover ( $\mathrm{Fr}$ ) and the surface temperature (Ts). Both variables were correlated for each type of land cover and eco-region. $\mathrm{Fr}$ and Ts are inversely associated and soil cover and different eco-regions showed changes in the line trend between $\mathrm{Fr}$ and $\mathrm{T}$ due to changes in the percentage 
and type of coverage, the rainfall gradients and vegetation structure. The mean values of $\mathrm{Fr}$ and FE for cover type and eco-regions, were processed using the analysis of variance and the multiple comparison test of Fisher. Statistically significant differences were found in Fr and FE according to type of land cover and eco-region and there is a nonlinear relationship between Fr and FE. Finally, a map was generated showing the spatial distribution of FE.

Keywords: evaporative fraction, eco-regions, MODIS, heterogeneity.

E.S. Luna Toledo y P.I. Figuerola: Universidad Nacional de Chilecito, 9 de Julio 22, 5360 Chilecito, La Rioja, Argentina. E.G. Abril y A.C. Ravelo: CREAN, Facultad de Ciencias Agropecuarias, UNC, CC 509, 5000 Córdoba, Argentina. Correspondencia a: etoledo@undec.edu.ar

\section{INTRODUCCION}

El monitoreo de la evapotranspiración (ET) aporta al conocimiento del ciclo hidrológico y a la valoración del estrés hídrico que afecta a los agroecosistemas a escala local, regional y continental (Allen, 2005). Diferentes métodos han sido propuestos para estimar la ET a distintas escalas espaciales, desde plantas individuales (flujo de savia, porómetros, lisímetros) a lotes agrícolas (balances hídrico y energético, covarianza del torbellino, bowen, escintillómetro) y a nivel de paisajes como el balance hídrico de cuencas (Wang et al., 2006). Sin embargo, estas técnicas convencionales proveen mediciones puntuales difíciles de extrapolar debido a la heterogeneidad y naturaleza dinámica de los procesos de transferencia de flujos (Rana \& Katerji, 2000).

El centro-noroeste de la Argentina presenta vastas zonas disímiles de vegetación (Cabrera, 1976; Burkart et al., 1999; Olson et al., 2001) y fuertemente alteradas por el avance de la agricultura y la ganadería (Boletta et al., 2006; Cagnolo et al., 2006), constituyendo así, paisajes heterogéneos difíciles de caracterizar mediante metodologías de estimación puntual de la ET. Los datos obtenidos por teledetección pueden ser utilizados para estimar la ET sobre amplias zonas con esas características. La ecuación de Priestley \&Taylor (1972) es una de las expresiones más conocidas para estimar la ET y tiene la ventaja de que todos sus términos pueden ser calculados utilizando información satelital (Batra et al., 2006).

La generación de imágenes por teledetección y su análisis digital ha permitido la caracterización de los patrones de uso del agua en diferentes ecosistemas, la determinación del uso consuntivo en agricultura, el ajuste de coeficientes de cultivos, la construcción de modelos de balance hidrológico y el monitoreo de cambio climático (Li et al., 2009; Glenn et al., 2010).

Una forma de cuantificar la partición de la energía disponible en un área $\left(A=R_{N}-G\right.$, donde $R_{N}$ : radiación neta y G: flujo de calor del suelo) a través de técnicas de teledetección es mediante el cálculo de la fracción evaporativa ( $F E=E T / A)$, que representa la energía usada para el proceso de ET (Brutsaert \& Sugita, 1992). La ventaja de usar FE resulta de su regularidad y constancia en días de cielo despejado (Shuttleworth et al., 1989). En este sentido, se puede admitir que su valor instantáneo representa satisfactoriamente un valor medio diurno (Mendonça et al., 2011).

El objetivo de este trabajo fue obtener la distribución de los valores de la fracción evaporativa (FE) para diferentes regiones agrícolas y naturales del centro-noroeste de la Argentina, utilizando la temperatura de superficie $\left(T_{s}\right)$ y la fracción de cobertura de suelo $(\mathrm{Fr})$ obtenidas por sensores remotos.

\section{MATERIALES Y METODOS}

\section{Descripción del área de estudio}

El área de estudio comprende una superficie superior a los $260.000 \mathrm{~km}^{2}$, que incluye porciones de las provincias de Santiago del Estero, Tucumán, Catamarca, La Rioja, Córdoba, Santa Fe y Chaco (República Argentina) (Figura1). La región posee veranos cálidos y húmedos, con un gradiente climático de este-oeste que varía de húmedo a sub-húmedo, semiárido, árido y desértico, excepto la porción norte del Chaco serrano. En el área se encuentran va- 
rias ecorregiones que comprenden: Monte, Chaco (seco, húmedo y serrano) y Espinal (Burkart et al., 1999; Olson et al., 2001, Omernik, 2004).

\section{Datos satelitales y procesamiento.}

La estimación de las variables Fr y FE se realizó utilizando datos de imágenes del sensor MODIS (Moderate Resolución Imaging Spectroradiometer) a bordo de la plataforma TERRA (EOS AM-1). Estas imágenes se encuentran calibradas y disponibles en forma gratuita (NASA, 2012a).La temperatura de superficie y el índice de vegetación de diferencia normalizada (NDVI) fueron obtenidas de las variables denominadas MOD11A2 y MOD13A2 correspondientes a diciembre de 2010. Esta fecha fue seleccionada por su representatividad de condiciones ambientales cálidas y húmedas. El área de estudio (Figura 1) fue seleccionada teniendo en cuenta dos criterios según requerimientos del método triangular (Jiang \& Islam 1999): (1), que la región presente un amplio rango de variación de cobertura vegetal/humedad del suelo; y (2), que sea una zona relativamente llana para que las diferencias en la temperatura de superficie resulten de la cobertura vegetal y no de las diferencias de altitud. Para cumplir con ambos criterios se seleccionó un área usando dos imágenes de MODIS (H12V11 y H12V12), que abarcan amplios rangos de cobertura, heterogeneidad de vegetación y clima (Figura 1). Las zonas montañosas fueron eliminadas del análisis mediante el uso de una máscara prepara- da a partir de un modelo de elevación digital Aster Global DEM V2 (NASA, 2012b).

El procesamiento de las imágenes MOD11A2 y MOD13A3 comprendió las siguientes etapas: importación a formato .img, construcción del mosaico H12V11/12, re-proyección a latitud/longitud, utilización del factor de escala, superposición de vectores, filtrado de valores incorrectos, análisis de matrices y obtención de NDVI y temperatura de superficie. Estos procedimientos se llevaron a cabo con el programa ERDAS (ERDAS, 2011).

\section{Fracción de la cobertura de suelo (Fr) y tempe- ratura de superficie $\left(T_{s}\right)$}

La Fr es propuesta por Tang et al. (2010) y reemplaza al NDVI (Tucker, 1979; Huete et al., 1999) en el espacio triangular que define la relación entre la temperatura de superficie y un índice de vegetación $\left(T_{\mathrm{s}}-\mathrm{IV}\right)$.

La ventaja principal de Fr es que mejora la representación de la proporción de suelo y vegetación para un área determinada. Se calcula como:

$\mathbf{F r}=\left[\left(\mathbf{N D V I}-\mathbf{N D V I}_{\min \amalg}\right) /\left(\mathbf{N D V I}_{\max }-\mathbf{N D V I}_{\min \boxminus}\right]^{2}\right.$ (1)

La $\mathrm{T}_{\mathrm{s}}$ es derivada de datos del infrarrojo térmico (Wan \& Li, 1997; Justice et al., 1998), utilizando el algoritmo Split-Window (Wan, 1999).

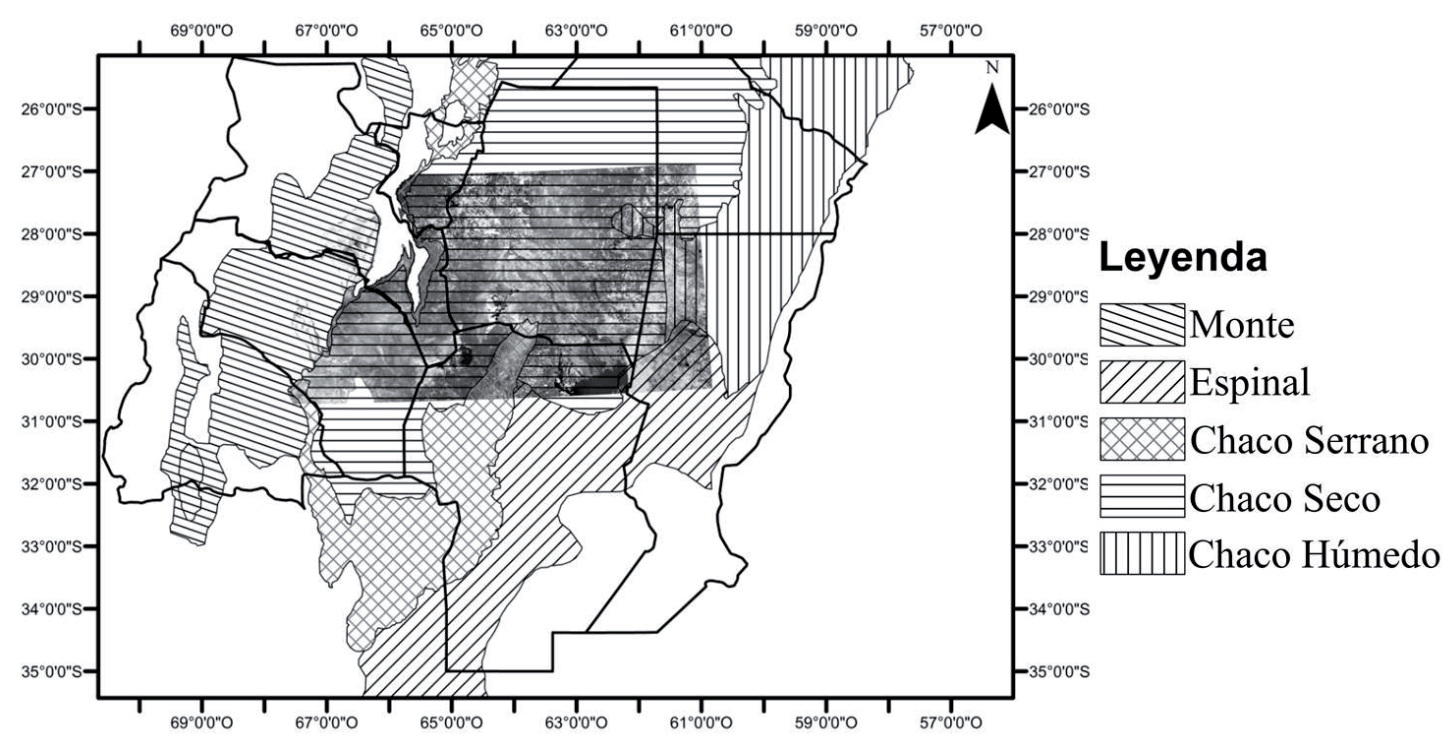

Figura 1. El área de estudio es la región sombreada que considera a ecorregiones del centro-noroeste de Argentina. 


\section{Componentes de la fracción evaporativa (FE)}

La fracción evaporativa es definida de la siguiente manera:

$\mathbf{F E}=\boldsymbol{\Phi}\left[\frac{\mathbf{s}}{\mathbf{s}+\gamma}\right]$

donde $\Phi$ es el parámetro de Jiang \& Islam (1999), s es la pendiente de la curva de presión de vapor de saturación y $\gamma$ es la constante psicrométrica; estos parámetros fueron obtenidos a partir de las imágenes MODIS (Tang et al., 2010).

\section{Construcción del espacio $\mathrm{T}_{\mathrm{s}}-\mathrm{Fr}$}

Los valores de Fr fueron calculados (ecuación 1) para cada píxel y graficados en función de versus los valores de $T_{s}$ en un diagrama de dispersión para toda el área de estudio.

Las siguientes consideraciones fueron tenidas en cuenta al construir el espacio $\mathrm{T}_{\text {s }}$ - Fr: (a), la Fr es calculada por píxeles de NDVI entre 0 (sin cobertura vegetal) y 1 (cobertura vegetal completa), y (b), los pixeles de Fr que no tenían su correspondiente valor de temperatura fueron eliminados.

Una vez construido el espacio $T_{s}-F r$ queda delimitado un triángulo, cuyos bordes se obtuvieron usando el algoritmo de determinación automática de bordes de Tang et al. (2010), eliminando así la subjetividad en la delimitación. Ésta es importante para eliminar relaciones entre pixeles que no cumplan con los supuestos del método usado.

\section{Cálculo del parámetro $\Phi$}

El método triangular relaciona $T_{s}$ y un índice de vegetación y permite estimar ET. Jiang \& Islam $(1999,2001)$ han aplicado este método para una extensa área heterogénea, y Petropoulos et al. (2009) han realizado una revisión completa de esta metodología. El parámetro $\Phi$ en la ecuación (2) es similar al parámetro $\alpha$ de la expresión de evapotranspiración potencial de Priestley-Taylor (Brutsaert, 1982), pero en este caso tiene en cuenta un amplio rango de humedad del suelo en la que $\Phi$ varía entre 0 y 1,26 , y en el cual están incluidos los efectos complejos de las resistencias que tiene lugar en el proceso de evapotranspiración. Además, cubre un amplio rango de condiciones evaporativas y no requiere datos atmosféricos ni de superficie.

El parámetro $\Phi$ se calculó usando un elevado número de pixeles ( 260.000) de $T_{s}-F r$ que permi- tieron definir el límite seco y húmedo en el espacio triangular (Jiang \& Islam, 1999; Tang et al., 2010).

\section{Selección de tipos de cobertura usando Google Earth (2010)}

La metodología de selección de los tipos de cobertura consistió en la identificación visual de áreas homogéneas (>1 km²) naturales o implantadas, mediante el uso de imágenes satelitales de alta resolución del programa Google Earth. Esta fuente de información utiliza imágenes del satélite QuickBird, y resulta adecuado en la identificación y mapeo de coberturas terrestres (Clark et al., 2010). Así, se definieron cinco tipos de cobertura: agrícola, árboles, arbustos, pastizales y suelo desnudo. Se seleccionaron 20 muestras por tipo de cobertura en cada ecorregión, con un total de 340 muestras en el área de estudio. No todas las ecorregiones presentaron todos los tipos de cobertura (p. e., el tipo de cobertura árboles, está ausente en la ecorregión monte). Las áreas identificadas a través de Google Earth fueron convertidas en vectores (con sus respectivos valores de latitud y longitud) con el fin de usarlas como archivo de recorte en las variables Fr y FE obtenidas desde imágenes MODIS, como se describió anteriormente.

Debido a la heterogeneidad de fechas con las que trabaja Google Earth, y con el objetivo de corroborar la existencia de los tipos de cobertura utilizados para el año 2010, fueron confrontados con imágenes del sensor Landsat TM para dicho año (Path/row: 231/79-80-81; 230/79-80-81; 229/79-8081 y 228/79-80-81).

\section{Análisis estadístico}

Los valores medios de las variables $\mathrm{Fr}, \mathrm{T}_{\mathrm{s}}$ y $\mathrm{FE}$ fueron obtenidos por cada tipo de cobertura y por cada ecorregión. La Fr y T fueron relacionadas usando el análisis de correlación (coeficiente de Pearson).

Las medias de las variables Fr y FE por tipos de cobertura y ecorregiones fueron tratadas estadísticamente mediante un análisis de la varianza con un nivel de significación de 0,05. Los resultados fueron comparados mediante el test de comparaciones múltiples LSD (mínima diferencia significativa) de Fisher (Balzarini et al., 2008).

El análisis estadístico de los datos se realizó mediante el programa InfoStat (Di Rienzo et al., 2012). 


\section{RESULTADOS Y DISCUSION}

\section{Fracción de cobertura de suelo y temperatura de superficie}

Las áreas con valores altos de Fr se encuentran asociadas con valores bajos de $T_{s}$. La relación no siempre es lineal, ya que varía para las diferentes ecorregiones y tipos de cobertura. Existen numerosos trabajos que asocian estas variaciones con el tipo de cobertura vegetal, la humedad del suelo, el albedo, la resistencia estomática y principalmente con la ET (Goward et al., 1985; Nemani et al., 1989; Price, 1990; Prihodko \& Goward, 1997; Jiang \& Islam, 1999, 2001; Sandholt et al., 2002; Goward, 2002, Tang et al., 2010).

El coeficiente de correlación ( $r$ ) entre $\mathrm{T}_{\mathrm{s}}$ y $\mathrm{Fr}$ brinda información del grado de asociación entre ambas variables. Un valor bajo del coeficiente indica una fuerte resistencia al cambio de temperatura (inercia térmica) cuando existe una variación de Fr, y es atribuido a altos valores de contenido de humedad en el suelo (Prihodko \& Goward, 1997).

Los diferentes tipos de ambientes ocupan distintos espacios, de acuerdo al método triangular de Jiang and Islam (1999, 2001), y por otra parte, el balance de energía en cada ecorregión se puede interpretar según la posición relativa que ocupan en dicho espacio triangular. La Figura 2 muestra la relación $T_{s}-F r$ de los distintas tipos de cobertura y ecorregiones. En general, las ecorregiones que muestran altos desvíos con respecto al valor medio de Fr y tienen un marcado gradiente de precipitación, tienden a mostrar una buena relación entre $T_{s}$ y Fr (por ej. Fig. 2c). Por el contrario, si se mantienen valores de coberturas vegetales muy bajas o muy altas la relación entre $T_{s}$ y Fr tiende a disminuir (por ej. Fig. 2a y 2e), incluso cuando se trata de una ecorregión que no presenta limitaciones hídricas.

El coeficiente de correlación entre la $\mathrm{T}_{\mathrm{s}}$ y la $\mathrm{Fr}$ que incluye todas las ecorregiones es de $-0,71$; se observa una correlación negativa, coincidiente con lo encontrado por otros autores (Price, 1990; Gillies \& Carlson, 1995; Prihodko \& Goward, 1997; Sandholt et al., 2002; Kim et al., 2005; Carlson, 2007; Tang et al., 2010). El aspecto triangular de la relación $T_{s}-F r$ implica que pequeñas variaciones de $\operatorname{Fr}(\Delta \operatorname{Fr} \sim 0,1)$ en el extremo de coberturas completas $(F r \sim 1)$ se asocian a situaciones de inercia de $\mathrm{T}_{\mathrm{s}}\left(\Delta \mathrm{T}_{\mathrm{s}} \sim 10 \mathrm{~K}\right)$; mientras que en el otro extremo del espacio, donde la proporción de suelo desnudo (Fr 0) es alta, se observa que a pequeñas variaciones de $\operatorname{Fr}(\Delta \mathrm{Fr} \sim 0,1), T_{s}$ muestra una mayor sensibilidad $\left(\Delta \mathrm{T}_{\mathrm{s}} \sim 30 \mathrm{~K}\right)$. En este último caso la ET real es baja y una elevada proporción de la energía es utilizada para calentar la superficie, aumentando $\mathrm{T}_{\mathrm{s}}$ (Han et al., 2006). Por lo tanto, pequeñas variaciones de la cobertura de suelo $(\Delta \mathrm{Fr} \sim 0,1)$ afectan más significativamente a la temperatura de superficie en áreas con $\mathrm{Fr} 0$ que con $\mathrm{Fr} \sim 1$.

El contenido de humedad del suelo posee un importante rol en el espacio triangular (Price, 1990; Carlson et al., 1995). Las ecorregiones con suelos húmedos, tales como los encontrados en Chaco Húmedo (entre 1300 y 700 mm/año de precipitación en sentido este a oeste), Espinal (entre 1200 y

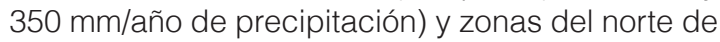
Chaco Serrano (>750 mm/año de precipitación), mantienen una inercia térmica. Por el contrario, las ecorregiones con déficit hídrico como Monte $(<200$ mm/año de precipitación), Chaco Seco (entre 700

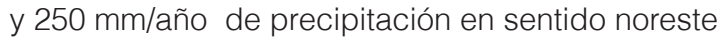
a suroeste) y porciones del Chaco Serrano muestran una mayor variación en su temperatura. Este comportamiento es explicado por Chuvieco Salinero (2008), para otros ambientes, basado en el alto calor específico y conductividad térmica que el agua le otorga a suelos húmedos.

\section{Relación entre las variables $\mathrm{T}_{\mathrm{s}}$ y Fr en las distin- tas ecorregiones}

Se obutuvieron los valores medios y sus respectivos desvíos estándar para las variables $T_{s}$ y Fr y se analizó la relación entre ambas para los distintos tipos de coberturas por ecorregiones:

Monte: En el tipo de cobertura agrícola existe una correlación negativa alta entre las $T_{s}-F r\left(r_{=}-\right.$ $0,75)$, debido principalmente al amplio rango de variación encontrado en la variable Fr para valores discretos de $T_{s}$ (Figura 2a). La agricultura en esta ecorregión se desarrolla únicamente bajo riego, y como consecuencia de esto, existe poca variación de la temperatura de superficie $\left(\mathrm{T}_{\mathrm{s}}\right)$ en zonas cultivadas. En suelo desnudo, el valor medio de $\mathrm{Fr}$ es prácticamente $0\left(\mathrm{Fr}_{=} 0,01 \pm 0,002\right)$, por lo que la relación con $T_{s}$ es baja $\left(r_{=}-0,37\right)$ (Figura 2a). A su vez, arbustos presenta un coeficiente de correlación moderado $\left(r_{=}-0,68\right)$ y muestra una fuerte variación de las temperaturas en un pequeño rango de variación de la Fr (Figura 2a). Esta ecorregión se caracteriza por presentar valores bajos de Fr y valores altos de $T_{s}$, ocupando el extremo superior izquierdo en el espacio T $\mathrm{s}$-Fr (Figura 2a).

Chaco Seco: Es la ecorregión más heterogénea del área de estudio, con un gradiente elevado de precipitaciones desde noreste al suroeste (Burkart et al., 1999). En árboles ocurre una variación importante de $\mathrm{Fr}$ con respecto a su valor medio $(\mathrm{Fr}=0,37 \pm 1,5)$ y permite observar una correlación 

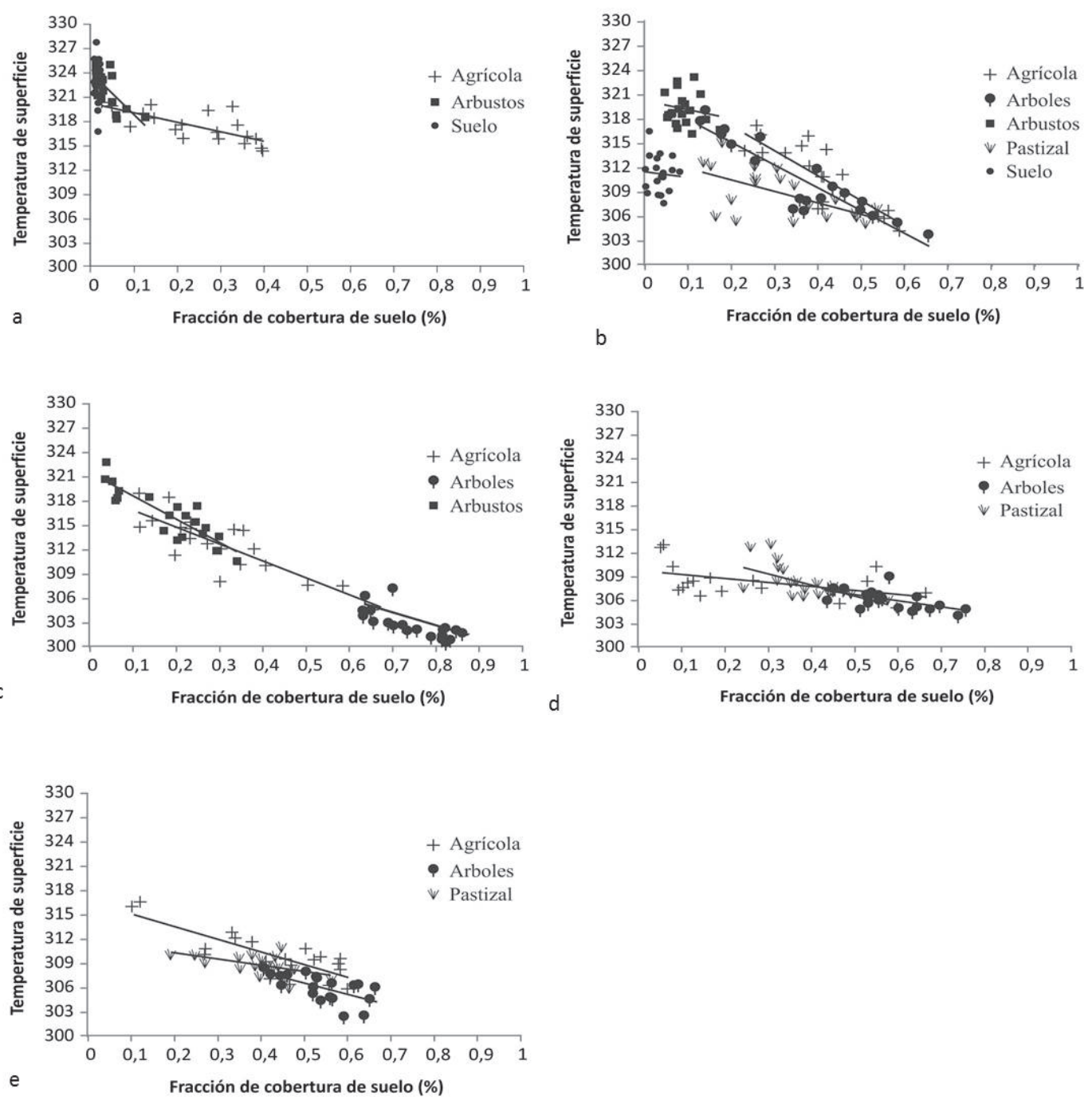

Figura 2. Gráfico de dispersión $T_{s}-F r$ para distintos tipos de cobertura por ecorregión. a) Monte; b) Chaco seco; c) Chaco serrano; d) Chaco húmedo; e) Espinal.

alta con $T_{s}\left(r_{=}-0,83\right)$ (Figura 2b). El tipo de cobertura agrícola presenta un coeficiente de correlación similar al anterior $\left(r_{-}-0,82\right)$, pero muestra una variación de $\mathrm{Fr}$ menor $(\mathrm{Fr}=0,40 \pm 0,11)$. El tipo de cobertura arbustos muestra una relación lineal muy baja $\left(r_{-}-0,16\right)$ entre las variables analizadas. La variación de temperatura en este tipo de cobertura no está explicada por los cambios en los valores de $\operatorname{Fr}(\mathrm{Fr}=0,09 \pm 0,03)$. En suelo desnudo no existe relación entre las variables analizadas (Figura $2 b)$, ya que $\mathrm{Fr}$ es muy cercana a $0(\mathrm{Fr}=0,04 \pm 0,02)$ y la relación es casi nula. En esta tipo de cobertura predominan ambientes de salinas, por lo tanto disminuciones o aumentos de la temperatura de superficie están asociados a cambios en el con- tenido de humedad de los suelos y a aumentos o disminuciones en su albedo por la alta reflectancia de la sal (Chaney et al., 1977).

Chaco Serrano: Esta ecorregión posee un marcado gradiente pluviométrico en sentido norte-sur desde los 900 a los 450 mm (Naumann, 2006). En el norte, las sierras conforman una barrera orográfica a los vientos húmedos del anticiclón del Atlántico sur (Burkart et al., 1999); mientras que hacia el sur, las cubiertas vegetales son menos densas y desaparecen algunos estratos en la vegetación, y los cambios en las coberturas del suelo se ven reflejados en cambios en la temperatura de superficie. El coeficiente de correlación es alto para arbustos $(-0,88)$ y agrícola $(-0,84)$ (Figura 2 c). El tipo 
de cobertura árboles tiene un alto coeficiente de correlación de $-0,74$, y presenta un elevado valor medio de la $\operatorname{Fr}\left(\mathrm{Fr}_{=} 0,75 \pm 0,08\right)$ y de poca variación (Figura 2c).

Chaco Húmedo: Los tipos de cobertura encontrados en esta ecorregión -agrícola, árboles y pastizales- presentan coeficientes de correlación moderados $(r-0,49 ;-0,57$ y $-0,59$ respectivamente) (Figura 2d) que pueden estar relacionados con el alto contenido de humedad del suelo en estas ecorregiones, que le confiere una elevada inercia térmica, lo cual coincide con lo presentado por Prihodko \& Goward (1997).

Espinal: Los tipos de cobertura árboles y pastizales presentaron una asociación moderada entre las variables (Figura 2e), con valores de correlación de -0,63 y -0,51, respectivamente. Estos coeficientes están determinados por el alto contenido de humedad de los suelos de esta porción del Espinal, que incluye los humedales de la laguna Mar Chiquita y los pantanos del río Dulce (Morello, 1959; Cabrera, 1976; Daniele \& Natenzon, 1994). El tipo de cobertura agrícola presenta una relación elevada entre las variables (Figura 2e) en comparación con otras ecorregiones, debido a las extensas áreas cultivadas.

\section{Análisis comparativo de los valores medios de Fr y FE}

En las Tablas 1 y 2 se presentan los valores medios y los resultados del test de comparaciones múltiples LSD de Fisher $(p=0,05)$ para la Fr y FE en cada tipo de cobertura.

Análisis de los valores medios de Fr por tipos de cobertura y ecorregiones

Agrícola: No se encontraron diferencias significativas en la $\mathrm{Fr}$ media para esta cobertura entre las ecorregiones Espinal $(\mathrm{Fr}=0,43)$ y Chaco Seco $(\mathrm{Fr}=0,40)$. Las ecorregiones Monte, Chaco Húmedo y Chaco Serrano no presentaron diferencias significativas en su media en este tipo de cobertura (Tabla 1). El Chaco Serrano y Chaco Seco no muestran diferencias significativas en sus valores de $\operatorname{Fr}(0,31$ y 0,40 respectivamente. En el Chaco Húmedo el tipo de cobertura agrícola está representada por el comienzo de ciclo de cultivos estacionales que presentan un valor bajo de NDVI, por lo que el valor de Fr para la fecha de estudio se muestra relativamente bajo en esta ecorregión.

Árboles: En el Chaco Seco y el Chaco Serrano se presentan los valores medios más bajos y más altos de $\mathrm{Fr}(\mathrm{Fr}=0,37$ y 0,75$)$ respectivamen-
Tabla 1. Valores medios y test de comparaciones múltiples LSD de Fisher $(p=0,05)$ para la fracción de cobertura de suelo. El superíndice indica la significancia para tipos de cobertura entre ecorregiones y el subíndice la significancia para tipos de cobertura en una misma ecorregión. Una letra en común indica que no son significativamente diferentes $(p<0,05)$

\begin{tabular}{|c|c|c|c|c|c|}
\hline \multicolumn{6}{|c|}{ Fracción de Cobertura (entre 0 y 1) $(\alpha=0,05)$} \\
\hline & Monte & $\begin{array}{c}\text { Chaco } \\
\text { seco }\end{array}$ & Espinal & $\begin{array}{l}\text { Chaco } \\
\text { húmedo }\end{array}$ & $\begin{array}{l}\text { Che } \\
\text { serr }\end{array}$ \\
\hline Árboles & --- & $0,37 \underset{a}{a}$ & $0,54_{a}^{b}$ & $0,58{ }_{a}^{b}$ & $0,75_{a}^{c}$ \\
\hline Agrícola & $0,23{ }_{a}^{a}$ & $0,40{ }_{a}^{b c}$ & $0,43_{c}^{c d}$ & $0,29{ }_{b}^{a}$ & $0,31_{b}^{b}$ \\
\hline Pastizales & --- & $0,32_{a}^{a}$ & $0,40_{b}^{a}$ & $0,37_{b}^{a}$ & --- \\
\hline Arbustos & $0,04_{b}^{a}$ & $0,09 b_{b}^{b}$ & --- & --- & $0,18{ }_{c}^{c}$ \\
\hline $\begin{array}{l}\text { Suelo } \\
\text { desnudo }\end{array}$ & $0,01_{c}^{a}$ & $0,04_{a}^{b}$ & --- & --- & --- \\
\hline
\end{tabular}

Tabla 2. Valores medios y test de comparaciones múltiples LSD de Fisher $(p=0,05)$ para la fracción evaporativa. El superíndice indica la significancia para tipos de cobertura entre ecorregiones y el subíndice la significancia para tipos de cobertura en una misma ecorregión. Una letra en común indica que no son significativamente diferentes $(p<0,05)$.

\begin{tabular}{|c|c|c|c|c|c|}
\hline \multicolumn{6}{|c|}{ Fracción evaporativa (entre 0 y 1) $(\alpha=0,05)$} \\
\hline \multirow{7}{*}{$\begin{array}{l}\text { Árboles } \\
\text { Agrícola } \\
\text { Pastizales } \\
\text { Arbustos } \\
\text { Suelo } \\
\text { desnudo }\end{array}$} & & Chaco & & Chaco & Chaco \\
\hline & Monte & seco & ES & húmedo & serrano \\
\hline & --- & 0,64 a & $0,77^{b}$ & $0,78^{b}$ & $0,87^{c}$ \\
\hline & $0,44_{a}^{a}$ & $0,63_{a}^{b c}$ & $0,66_{c}{ }_{c}^{a} d$ & $0,72_{b}^{a} a$ & $0,59_{b}^{a} b$ \\
\hline & --- & $0,71_{a}^{a}$ & $0,69_{b}^{a}$ & $0,71_{b}^{a}$ & \\
\hline & $0,28_{b}^{a}$ & $0,38_{b}^{a} b$ & --- & --- & $0,47_{\mathrm{c}}^{\mathrm{c}}$ \\
\hline & $0,21_{c}^{a}$ & $0,63_{a}^{b}$ & --- & --- & --- \\
\hline
\end{tabular}

te, con diferencias significativas (Tabla 1). Estas diferencias se deben a que los bosques del Chaco Seco están fuertemente degradados (Brown et al., 2006). La disponibilidad de precipitaciones es mayor en el Chaco Serrano, existiendo también múltiples estratos vegetales en algunas zonas de esta ecorregión (Cagnolo et al., 2006).El tipo de cobertura árboles en Espinal $(\mathrm{Fr}=0,54)$ y Chaco Húmedo $(\mathrm{Fr}=0,58)$ presentan valores intermedios sin diferencias significativas. El importante avance de la agricultura en estas ecorregiones ha dejado fragmentos pequeños de la cobertura boscosa original (Burkart et al., 1999).

Arbustos: Se presentan diferencias significativas en tres ecorregiones: Monte, Chaco Seco y Chaco Serrano. Los valores de $\mathrm{Fr}$ se mantienen bajos, alcanzando los valores mínimos en la ecorregión de Monte $(\mathrm{Fr}=0,04)$, donde la vegetación es una estepa arbustiva muy abierta y donde hay una alta proporción de suelo desnudo (Burkart et al., 1999; Brown et al., 2006). Los arbustos de Chaco Seco $(\mathrm{Fr}=0,09)$, principalmente localizados en la subregión denominada Chaco árido, incrementan su Fr levemente. La zona está caracterizada por fuertes procesos de degradación de la tierra 
(Brown et al., 2006). Finalmente en Chaco Serrano este tipo de cobertura está asociado a la parte más austral de la ecorregión, en donde las precipitaciones disminuyen y el estrato arbóreo va desapareciendo. Esta ecorregión presenta los valores más altos para arbustos.

Pastizales: No hay diferencias significativas entre las $\mathrm{Fr}$ del Espinal $(\mathrm{Fr}=0,40)$ y Chaco Húmedo $(\mathrm{Fr}=0,37)$, pero si existen diferencias entre estos dos y el Chaco Seco $(F r=0,32)$. Esto puede ser explicado por las diferencias en precipitaciones de las dos primeras con respecto a la última ecorregión, que configuran tipos y estructuras de pastizales distintos.

Suelo desnudo: Esta tipo de cobertura solo se encuentra en el Monte $(\mathrm{Fr}=0,01)$ y Chaco Seco $(\mathrm{Fr}=0,04)$ y ambas ecorregiones presentan diferencias significativas. En Chaco Seco esta fundamentalmente ligada a la presencia de salinas y suelos arados, mientras que en el Monte está asociada a zonas bajas no necesariamente salinas pero con menores precipitaciones ( $<150 \mathrm{~mm}$ anuales).

Análisis de los valores medios de FE por tipos de cobertura y ecorregiones

El análisis de los valores de FE (Tabla 2) muestra diferencias en la variable según el tipo de cobertura analizada para cada ecorregión.
En los valores medios de FE por ecorregión (sin discriminar tipos de cobertura) se encuentran diferencias significativas entre Monte ( $\mathrm{FE}=0,31)$, Chaco Seco $(F E=0,59)$, y Chaco Serrano ( $F E=0,65)$. En las ecorregiones Espinal $(F E=0,71)$ y Chaco Húmedo $(F E=0,74)$ no se encontraron diferencias significativas entre ellas para esta variable; además, fueron las que presentan los mayores valores medios de FE en el área de estudio. Estas diferencias son debidas a las variaciones en los tipos de cobertura y a las condiciones ambientales de cada una de las ecorregiones, y tienen un rol destacado la humedad del suelo.

Los valores medios de FE por tipos de cobertura (sin discriminar por ecorregiones): arbustos $(\mathrm{FE}=0,38)$ y suelo desnudo $(\mathrm{FE}=0,42)$ no mostraron diferencias significativas. Los tipos de cobertura agrícola ( $\mathrm{FE}=0,61)$, pastizales $(\mathrm{FE}=0,70)$ y árboles $(\mathrm{FE}=0,77)$ mostraron diferencias significativas entre sí y respecto a las dos anteriores. Estos resultados permiten inferir que disminuciones o cambios en el uso del suelo de árboles a agrícola, arbustos o suelo desnudo afectan significativamente al balance de energía de la ecorregión, disminuyendo la fracción de la energía utilizada en la ET, y por consiguiente aumentando el calor sensible.

La Figura 3 presenta la relación entre la Fr y FE para todas los tipos de cobertura y ecorregiones.

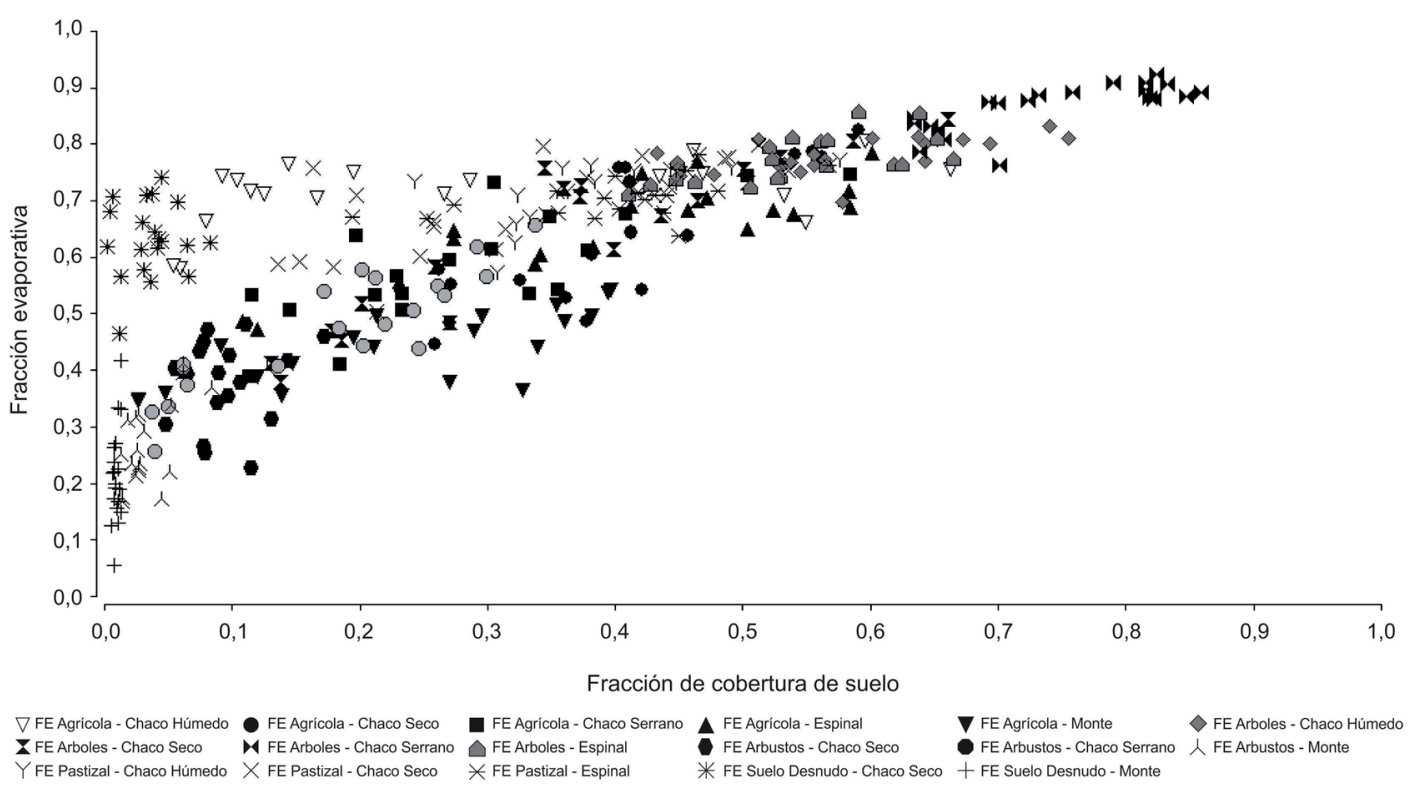

Figura 3. Diagrama de dispersión de fracción evaporativa (FE) y fracción de la cobertura (Fr) de suelo para ecorregiones y tipos de cobertura. 
Se observa gráficamente que para valores bajos de $\operatorname{Fr}(<0,4)$ existe una importante dispersión de la FE; en cambio, cuando Fr es superior a 0,4 la dispersión es menor, independientemente de las tipos de cobertura y ecorregiones.

\section{Zonas de características relevantes de FE}

La Figura 4 presenta una imagen de FE donde se destacan numéricamente algunas zonas que se analizan a continuación:

(1) Bolsón de Pipanaco. Posee valores bajos de FE y Fr. Se trata de una zona deprimida, desértica y endorreica, rodeada de grandes cordones montañosos que aíslan la zona de la llegada de masas de aire húmedo provenientes del Pacifico o del Atlántico y constituyen un paisaje con muy poca vegetación, alta proporción de suelos desnudos, alta insolación y baja precipitación (Bianchi y Bravo, 2008).

(2) Valle de Chilecito. En este valle se distingue un cinturón agrícola que posee valores altos de FE y Fr, rodeado de una zona de baja cobertura y densidad de vegetación. Es la región árida de mayor desarrollo olivícola y vitícola de la provincia de La Rioja.

(3) Zona del Chaco Seco en las provincias de La Rioja y Catamarca. Se trata de una zona sumamente degradada por sobrepastoreo de la ganadería y extracción forestal; presenta los valores más bajos de FE de toda la ecorregión del Chaco, y los arbustos son su cobertura vegetal predomi- nante (Brown et al., 2006).

(4) Zonas de desarrollo agrícola. Para la fecha de estudio, en este mosaico de cultivos se presentan diferentes etapas de crecimiento, que van desde el inicio a estadios medios, denotados por cambios espaciales en la Fr (Fritz et al., 2009). Los valores de FE también se muestran cambiantes, con valores entre 0,3 y 0,7 .

(5) Cuenca de la Laguna Mar Chiquita y del Río Dulce. Son zonas con alta humedad de suelo. En zonas inundables se encuentran valores bajos de Fr, baja $\mathrm{T}_{\mathrm{s}} \mathrm{y}$ valores altos de FE.

(6) Zonas del Bosque Chaqueño Santiagueño, bañados al norte del Río Salado y de Figueroa. Los bosques densos del centro-norte de la provincia de Santiago del Estero constituyen coberturas de vegetación con valores altos de Fr y de FE. El río Salado posee una serie de derrames principalmente sobre su margen derecha, como los Bañados de Figueroa (Ginzburg et al., 2005), que conforman un área en donde la evapotranspiración usa una gran proporción de la energía disponible.

(7) Zona agrícola y con vegetación natural. Esta zona constituye un mosaico de agricultura estacional, bosques y pastizales en el ecotono de Chaco Seco y Chaco Húmedo.

(8) Cuenca de Río Hondo. Esta vasta región agrícola (> 400.000 has), que se riega con aguas del embalse de Río Hondo, posee algunos de los más altos valores de FE de la ecorregión de Chaco Seco con valores altos de Fr.

\section{(9) Zona del Chaco Serrano en la provincia}

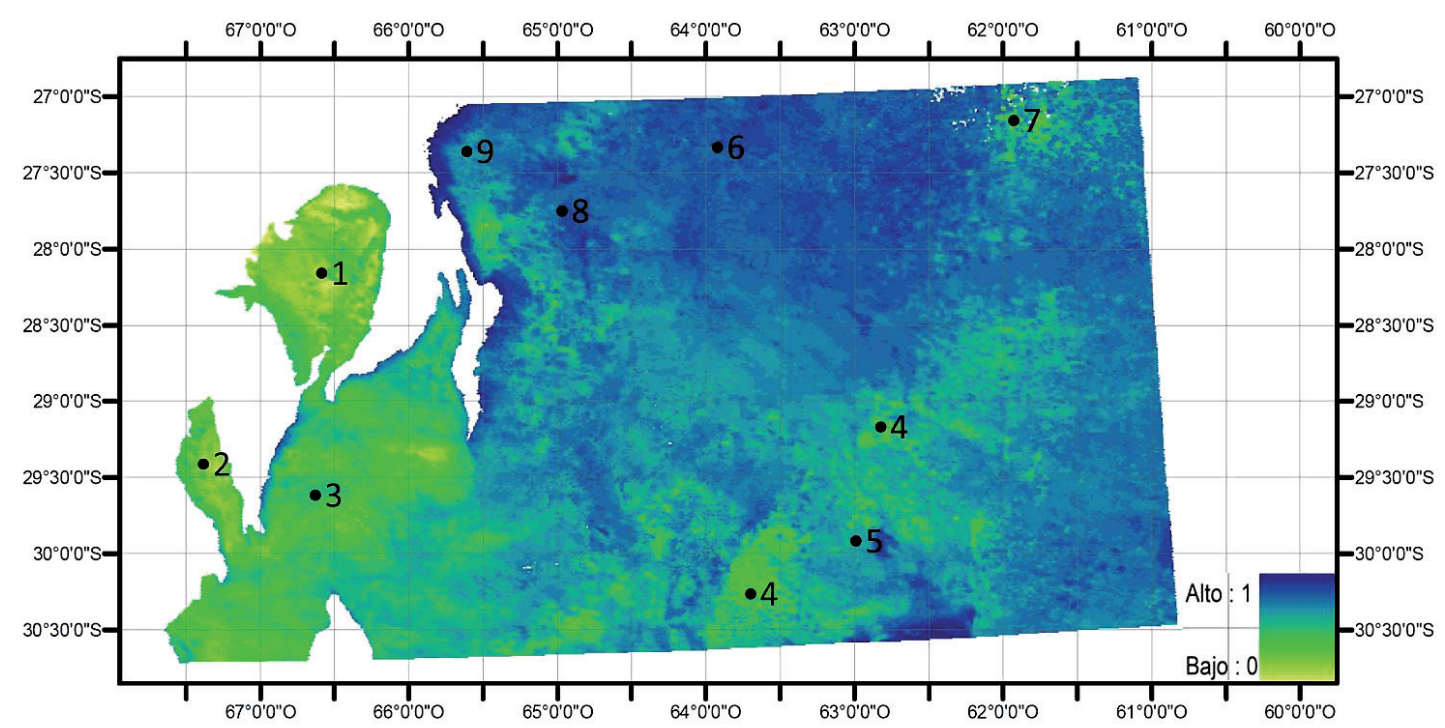

Figura 4. Imagen de la fracción evaporativa y zonas con características relevantes. 
de Tucumán. Posee una estructura densa de bosques, con múltiples estratos vegetales, limita con la selva de Yungas y presenta valores altos de $\mathrm{Fr}$ $(0,75)$ y $F E(0,87)$, los mayores de toda el área de estudio.

\section{CONCLUSIONES}

La energía disponible se utiliza en forma diferente en las ecorregiones según los tipos de vegetación y a las condiciones ambientales. En las ecorregiones húmedas se usa una gran proporción de la energía disponible en el proceso de evapotranspiración y mantienen una inercia térmica, mientras que las ecorregiones con déficit hídrico destinan esta energía al calor sensible.

El balance de energía es afectado por el tipo de uso del suelo y su cobertura. El cambio de cobertura arbórea a arbusto o a agrícola o a suelo desnudo disminuye la fracción de la energía utilizada en la evapotranspiración y el aumento de aquella usada en calor sensible.

La información aportada por imágenes satelitales permite un detallado análisis territorial y puede contribuir a la toma de decisiones para la planificación en el uso de la tierra a escala regional.

\section{AGRADECIMIENTOS}

AI D.G. Alejandro Barbeito por la preparación de las ilustraciones. Este trabajo fue financiado por el proyecto PICT-2007-00389 de la Agencia Nacional de Promoción Científica y Tecnológica.

\section{BIBLIOGRAFIA}

Allen, R. G.; M. Tasumi, A. Morse and R. Trezza, 2005. A Landsat-based energy balance and evapotranspiration model in Western US water rights regulation and planning. Irrigation and Drainage Systems. 19:251268.

Balzarini, M. G.; L. Gonzalez, M. Tablada, F. Casanoves, J. A. Di Rienzo y C.W. Robledo, 2008. InfoStat, Manual del Usuario, Editorial Brujas, Córdoba, Argentina.

Batra, N.; S. Islam, V. Venturini, G. Bisht and L. Jiang, 2006. Estimation and Comparison of Evapotranspiration from Multiple Satellites for Clear Sky Days. Remote Sensing of Environment. 103:1-15.

Bianchi, A. R. y G. C. Bravo, 2008. Ecorregión Norandina. Descripción, Subregiones, Agroecosistemas, Sistemas productivos y Cartografía regional. Ediciones Instituto Nacional de Tecnología Agropecuaria. 60 pp.
Boletta, P. E.; A. C. Ravelo, A. M. Planchuelo and M. Grilli, 2006. Assessing deforestation in the Argentine Chaco. Forest Ecology and Management. 228:108-114.

Brown, A.; U. Martinez Ortiz, M. Acerbi y J. Corcuera (eds.), 2006. La Situación Ambiental Argentina 2005. Fundación Vida Silvestre Argentina. Buenos Aires. 587 pp.

Brutsaert, W., 1982. Evaporation into the atmosphere: Theory, History and Applications. D.Reidel, Norwell, Mass. 299 pp.

Brutsaert, W. and M. Sugita, 1992. Application of self-preservation in the diurnal evolution of the surface energy budget to determine daily evaporation. Journal of Geophysical Research Letters. 97(D17):18377-18382.

Burkart, R.; N. O. Barbaro, O. R. Sanchez y D. Gomez, 1999. Eco-regiones de la Argentina. Administración de Parques Nacionales y Programa de Desarrollo Institucional Ambiental, Buenos Aires, 42 pp.

Cabrera, A. L., 1976. Regiones Fitogeográficas Argentinas. Enciclopedia argentina de agricultura y jardinería. Tomo II. Fascículo I. Acme, Buenos Aires. pp 1-85.

Cagnolo, L.; M. Cabido and G. Valladares, 2006. Plant species richness in the Chaco Serrano Woodland from central Argentina: Ecological traits and habitat fragmentation effects. Biological Conservation. 132:510519

Carlson, T., 2007. An overview of the "Triangle Method" for estimating surface evapotranspiration and soil moisture from satellite imagery. Sensors. 7:1612-1629.

Carlson, T. N.; W. J. Capehart and R. R. Gillies, 1995. A new look at the simplified method for remote sensing of daily evapotranspiration. Remote Sensing of Environment. 54:161-167

Chaney, J.; W. J. Quirk, S. H. Chow and J. Kornfied, 1977. A comparative study of the effect of albedo change on drought in semi-arid regions. Journal of the Atmospheric Science. 34:1366-1385

Chuvieco Salinero, E. 2008. Teledetección ambiental: La observación de la tierra desde el espacio. $3^{a}$ edición. Editorial Ariel Ciencia. Barcelona. 67-76 pp.

Clark, M. L.; T. M. Aide, H. R. Grau and G. Riner, 2010. A scalable approach to mapping annual land cover at 250 m using MODIS time series data: A case study in the Dry Chaco ecoregion of South America. Remote Sensing of Environment. 114:2816-2832.

Daniele, C. and C. Natenzon, 1994. Regiones Naturales de la Argentina. Draft map. Argentina National Parks Department, Buenos Aires, Argentina.

Di Rienzo. J. A.; F. Casanoves, M. G. Balzarini, L. González, M. Tablada Y C. W. Robledo, 2012. InfoStat versión 2012. Grupo InfoStat, FCA, Universidad Nacional de Córdoba, Argentina. URL <http://www.infostat. com.ar> Consultada el 27/12/2012. 
ERDAS, 2011. URL <http://www.erdas.com.ar/productos _imagine.htm> Consultada el 27/12/2012.

Fritz, S.; I. McCallum, C. Schill, C. Perger, R. Grillmayer, F. Achard, F. Kraxner, and M. Obersteiner. 2009. GeoWiki.Org: The Use of Crowdsourcing to Improve Global Land Cover. Remote Sensing. 1(3):345-354. URL <http://www.geo-wiki.org/> Consultada el 27/12/2012.

Gillies, R. R. and T. N. Carlson, 1995. Thermal remote sensing of surface soil water content with partial vegetation cover for incorporation into mesoscale prediction models. Journal of Applied Meteorology. 34:745-756.

Ginzburg, R.; J. Adámoli, P. Herrera y S. Torrella, 2005. Los humedales del chaco: Clasificación, inventario y mapeo a escala regional. Insugeo, miscelánea, San Miguel de Tucumán. 121-138.

Glenn, E.P.; P. L. Nagler and A. R. Huete, 2010. Vegetation index methods for estimating evapotranspiration by remote sensing. Surveys in Geophysics. 31:531-555.

Google Earth. 2012. <http://www.google.com/earth/ index.html> Consultada el 27/12/2012.

Goward, S. N.; Y. Xue and K. P. Czajkowski, 2002. Evaluating land surface moisture conditions from the remotely sensed temperature/vegetation index measurements. An exploration with the simplified simple biosphere model. Remote Sensing of Environment. 79:225-242.

Goward, S.; G. D. Cruickshanks and A. Hope, 1985. Observed relation between thermal emission and reflected spectral radiance of a complex vegetated landscape. Remote Sensing of Environment. 18:137-146.

Han, L.; P. Wang, H. Yang, S. Liu and J. Wang, 2006. Study on NDVI-Ts space by combining LAI and evapotranspiration. Science in China Series. 49 (D7): 747-754

Huete, A.; C. O. Justice and W. J. D. Van Leeuwen, 1999. MODIS Vegetation Index (MOD13), Algorithm Theoretical Basis Document (ATBD) Version 3.0, pp. 129

Jiang, L. and S. Islam, 1999. A methodology for estimation of surface evapotranspiration over large areas using remote sensing observations. Geophysical Research Letters. 26: (17) 2773-2776.

Jiang, L. and S. Islam, 2001. Estimation of surface evaporation map over southern Great Plains using remote sensing data. Water Resources Research. 37: (2) 329340.

Justice, D. H.; V. Salomonson, J. Privette, G. Riggs, A. Strahler, R. Lucht, R. Myneni, Y. Knjazihhin, S. Running, R. Nemani, E. Vermote, J. Townshend, R. Defries, D. Roy, Z. Wan, A. Huete, R. Van Leeuwen, R. Wolfe, L. Giglio, J. P. Muller, P. Lewis and M. Barnsley, 1998. The Moderate Resolution Imaging Spectro radiometer (MODIS): land remote sensing for global change research. IEEE Transactions on Geoscience and Remote Sensing. 36:1228-1249.

Kim, H. M.; B. K. Kim and K. S. You, 2005. A Statistic Co- rrelation Analysis Algorithm Between Land Surface Temperature and Vegetation Index. International Journal of Information Processing Systems. 1:102-106

Li, Z.; R. Tang, Z. Wan, Y. Bi, C. Zhou and B. Tang, 2009. A review of current methodologies for regional evapotranspiration estimation from remotely sensed data. Sensors. 9:3801-3853.

Mendonça, J. C.; E. Fernandes de Sousa, R. G. Bouhid André, B. Barbosa da Silva and N. J. Ferreiral, 2011. Assessment of evapotranspiration in North Fluminense Region, Brazil using MODIS products and Sebal algorithm. En Ayse Irmak (Ed). Evapotranspiration, remote sensing and modeling. Croatia. Published by InTech. Chapter 1:1-18 pp.

Morello, J. H., 1959. La provincia Fitogeográfica del Monte. Opera Lilloana II, Tucumán, Instituto Miguel Lillo, $155 \mathrm{pp}$.

NASA, 2012a. REVERB ECHO. EOSDIS NASA's Earth Observing System. Data and Information System. $<$ http://reverb.echo.nasa.gov/reverb> Consultada el 27/12/2012.

NASA, 2012b. ASTER global digital elevation map announcement. <http://asterweb.jpl.nasa.gov/gdem. asp> Consultada el 27/12/2012.

Naumann, M., 2006. Atlas del Gran Chaco Sudamericano. Sociedad Alemana de Cooperación Técnica (GTZ). ErreGé \& Asoc. Buenos Aires. 92 pp.

Nemani, R. R. And S. W. Running, 1989. Estimation of regional surface resistance to evapotranspiration from NDVI and thermal-IR AVHRR Data. Journal of Applied Meteorology. 28:276-284.

Olson, D.M.; E. Dinerstein, E. D. Wikramanayake, N. D. Burgess,G. V. N. Powell, E. C. Underwood, J. A. D’Amico, H. E. Strand, J. C. Morrison, C. J. Loucks, T. F. Allnutt, J. F. Lamoreux, T. H. Ricketts, I. Itoua, W. W. Wettengel, Y. Kura, P. Hedao and K. Kassem, 2001. Terrestrial ecoregions of the world: A new map of life on Earth. BioScience 51:933-938.

Omernik, J. M., 2004. Perspectives on the nature and definition of ecological regions. Environmental Management 34:S27-S38.

Petropoulos, G.; T. N. Carlson, M. J. Wooster and S. A. Islam, 2009. A review of Ts/VI remote sensing based methods for the retrieval of land surface energy fluxes and soil surface moisture. Progress in Physical Geography. 33:224-250.

Price, J. C., 1990. Using spatial context in satellite data to infer regional scale evapotranspiration. IEEE Transactions on Geoscience and Remote Sensing. 28:940948.

Priestley, C. H. B. and R. J. Taylor, 1972. On the Assessment of Surface Heat Flux and Evaporation Using Large-Scale Parameters. Monthly Weather Review. 
100:81-92.

Prihodko, L. and S. N. Goward, 1997. Estimation of air temperature from remotely sensed surface observations. Remote Sensing of Environment. 60:335-346

Rana, G. and N. Katerji, 2000. Measurement and estimation of actual evapotranspiration in the field under Mediterranean climate: a review. European Journal of Agronomy. 13:125-153.

Sandholt, I.; K. Rasmussen and J. Andersen, 2002. A simple interpretation of the surface temperature/vegetation index space for assessment of surface moisture status. Remote Sensing of Environment. 79:213-224

Shuttleworth, W. J.; R. J. Gurney, A. Y. Hsu and J. P. Ormsby, 1989. FIFE: The variation in energy partition at surface flux sites, IAHS Publ. 186:67-74.

Tang R. L.; Z. L. Li and B. H. Tang, 2010. An application of the Ts-VI triangle method with enhanced edges determination for evapotranspiration estimation from MODIS data in arid and semi-arid regions: Implemen- tation and validation. Remote Sensing of Environment 114:540-551.

Tucker, C. J., 1979. Red and photographic infrared linear combinations for monitoring vegetation. Remote Sensing of Environment, 8:127-150.

Wan Z., 1999. MODIS Land-Surface Temperature (LST ATBD) Version 3.3. Obtenido desde NASA MODIS WEB. <http://modis.gsfc.nasa.gov/data/atbd/atbd_ mod11.pdf> Consultada el 27/12/2012.

Wan Z. and Z. L. Li, 1997. A physics-based algorithm for retrieving land surface emissivity and temperature from EOS/MODIS data. IEEE Transactions on Geoscience and Remote Sensing. 35:980-996.

Wang, K.; Z. Li and M. Cribb, 2006. Estimation of evaporative fraction from a combination of day and night land surface temperatures and NDVI: A new method to determine the Priestley-Taylor parameter. Remote Sensing of Environment. 102: 293-305. 